\title{
Multiparametric MRI and auto-fixed volume of interest-based radiomics signature for clinically significant peripheral zone prostate cancer
}

\author{
Jeroen Bleker $^{1}$ (D) $\cdot$ Thomas C. Kwee $^{1} \cdot$ Rudi A. J. O. Dierckx ${ }^{1} \cdot$ Igle Jan de Jong ${ }^{2} \cdot$ Henkjan Huisman $^{3} \cdot$ Derya Yakar $^{1}$
}

Received: 5 July 2019 /Revised: 28 August 2019 / Accepted: 9 October 2019/Published online: 27 November 2019

(C) The Author(s) 2019

\begin{abstract}
Objectives To create a radiomics approach based on multiparametric magnetic resonance imaging (mpMRI) features extracted from an auto-fixed volume of interest (VOI) that quantifies the phenotype of clinically significant (CS) peripheral zone (PZ) prostate cancer $(\mathrm{PCa})$.

Methods This study included 206 patients with 262 prospectively called mpMRI prostate imaging reporting and data system 3-5 PZ lesions. Gleason scores $>6$ were defined as CS PCa. Features were extracted with an auto-fixed 12-mm spherical VOI placed around a pin point in each lesion. The value of dynamic contrast-enhanced imaging(DCE), multivariate feature selection and extreme gradient boosting (XGB) vs. univariate feature selection and random forest (RF), expert-based feature pre-selection, and the addition of image filters was investigated using the training (171 lesions) and test (91 lesions) datasets.

Results The best model with features from T2-weighted (T2-w) + diffusion-weighted imaging (DWI) + DCE had an area under the curve (AUC) of 0.870 (95\% CI 0.980-0.754). Removal of DCE features decreased AUC to 0.816 (95\% CI 0.920-0.710), although not significantly $(p=0.119)$. Multivariate and XGB outperformed univariate and RF $(p=0.028)$. Expert-based feature pre-selection and image filters had no significant contribution.

Conclusions The phenotype of CS PZ PCa lesions can be quantified using a radiomics approach based on features extracted from T2-w + DWI using an auto-fixed VOI. Although DCE features improve diagnostic performance, this is not statistically significant. Multivariate feature selection and XGB should be preferred over univariate feature selection and RF. The developed model may be a valuable addition to traditional visual assessment in diagnosing CS PZ PCa.

Key Points

- T2-weighted and diffusion-weighted imaging features are essential components of a radiomics model for clinically significant prostate cancer; addition of dynamic contrast-enhanced imaging does not significantly improve diagnostic performance.

- Multivariate feature selection and extreme gradient outperform univariate feature selection and random forest.

- The developed radiomics model that extracts multiparametric MRI features with an auto-fixed volume of interest may be a valuable addition to visual assessment in diagnosing clinically significant prostate cancer.
\end{abstract}

Keywords Machine learning $\cdot$ Magnetic resonance imaging $\cdot$ Prostatic neoplasms $\cdot$ Neoplasm grading

Electronic supplementary material The online version of this article (https://doi.org/10.1007/s00330-019-06488-y) contains supplementary material, which is available to authorized users.

Jeroen Bleker

j.bleker@umcg.nl

1 Medical Imaging Center, Departments of Radiology, Nuclear Medicine and Molecular Imaging, University Medical Center Groningen, University of Groningen, Hanzeplein 1, 9700 RB Groningen, The Netherlands
2 Department of Urology, University Medical Center Groningen, University of Groningen, Hanzeplein 1, 9700 RB Groningen, The Netherlands

3 Department of Radiology and Nuclear Medicine, Radboud University Medical Center, Geert Grooteplein Zuid 10, 6525 GA Nijmegen, The Netherlands 


$\begin{array}{ll}\text { Abbreviations } \\ \text { 2D LBP } & \text { Two-dimensional local binary pattern } \\ \text { ADC } & \text { Apparent diffusion coefficient } \\ \text { AUC } & \text { Area under the curve } \\ \text { CI } & \text { Confidence interval } \\ \text { CS } & \text { Clinically significant } \\ \text { DCE } & \text { Dynamic contrast-enhanced } \\ \text { DRE } & \text { Digital rectal examination } \\ \text { DWI } & \text { Diffusion-weighted imaging } \\ \text { GLCM } & \text { Gray level co-occurrence matrix } \\ \text { GLDM } & \text { Gray level dependence matrix } \\ \text { GLRLM } & \text { Gray level run length matrix } \\ \text { GLSZM } & \text { Gray level size zone matrix } \\ \text { H } & \text { High-pass filter } \\ \text { ISUP } & \text { International Society of Urological Pathology } \\ \text { L } & \text { Low-pass filter } \\ \text { LoG } & \text { Laplacian of Gaussian } \\ \text { mpMRI } & \text { Multiparametric magnetic resonance imaging } \\ \text { NGTDM } & \text { Neighboring gray tone difference } \\ & \text { matrix features } \\ \text { PCa } & \text { Prostate cancer } \\ \text { PI-RADS } & \text { Prostate imaging reporting and data system } \\ \text { PZ } & \text { Peripheral zone } \\ \text { RF } & \text { Random forest } \\ \text { ROC } & \text { Receiver operating curve } \\ \text { T2-w } & \text { T2-weighted imaging } \\ \text { TZ } & \text { Transition zone } \\ \text { VOI } & \text { Volume of interest } \\ \text { XGB } & \text { Extreme gradient boosting } \\ & \end{array}$

\section{Introduction}

Prostate cancer ( $\mathrm{PCa})$ is currently the most common cancer among men, and comprises approximately $20 \%$ of all cancers in the western world $[1,2]$. Although most patients with PCa can be successfully treated [3], it is still responsible for an estimated $10 \%$ of all male cancerrelated deaths in the western world. Early and accurate detection of clinically significant (CS) $\mathrm{PCa}$ is important to initiate treatment in a timely manner and improve patient outcome [3].

Current methods used for the detection of PCa vary per institution. Nevertheless, prostate-specific antigen (PSA) testing with digital rectal examination (DRE) followed by transrectal ultrasound (TRUS) biopsy is a widely used diagnostic algorithm. However, PSA testing suffers from a high number of false positives combined with a considerable number of false negatives [4]. The high falsepositive rate leads to unnecessary TRUS biopsies. Furthermore, TRUS biopsies also suffer from sampling errors (i.e., both false negatives and underestimation of the true Gleason grade) [5]. The diagnostic limitations of
PSA testing followed by TRUS biopsies lead to unnecessary patient discomfort, anxiety, and complications [6].

Multiparametric magnetic resonance imaging (mpMRI) has gained popularity as a non-invasive imaging technique for CS $\mathrm{PCa}$ detection and biopsy guidance that may overcome many of the shortcomings of the combination of PSA and TRUS alone [7-9]. Despite its potential, correct diagnosis of CS PCa based on mpMRI requires skill and experience. With the introduction of PI-RADS, and later PI-RADS v2, the diagnostic performance of radiologists has improved [8, 10]. Nevertheless, PIRADS v2 is by no means a perfect system. Radiologists still need extensive experience to correctly discriminate CS from non-CS tumors [11, 12], with the additional issue that some lesions are not visible on mpMRI [13, 14]. Computer-aided diagnosis (CAD) aimed to increase correct diagnosis; however, due to the use of a small group of handcrafted features, its success is dependent on expert knowledge [15]. Therefore, there is a need for new technology that improves CS PCa detection on mpMRI without expert knowledge dependency. The use of radiomics, which aims to extract relevant quantitative tumor features from imaging data that may be unperceivable by the human eye, may fill this void [16].

A limited number of studies already aimed to find such quantitative mpMRI radiomics features for CS PCa [17-19]. However, these previous studies suffered from several methodological shortcomings, including small sample sizes (as low as 30 patients), heterogeneous datasets mixing peripheral zone (PZ) with transition zone (TZ) tumors, manual delineation of tumor suspicious regions (which introduces observer dependency and decreases model generalization), and a very small number of initial quantitative features that were explored (as low as 10 features). Furthermore, no previous radiomics study investigated whether the use of dynamic contrast-enhanced (DCE, k-trans) sequences adds useful diagnostic information to a radiomics-based approach. Finally, no research has been performed on whether the multivariate-based diagnosis of CS PCa on mpMRI works better with multivariate feature selection and extreme gradient boosting (XGB) $[20,21]$ than the recommended univariate selection and random forest (RF) [22].

The aim of this study was to create a model based on mpMRI radiomics features extracted from an auto-fixed volume of interest (VOI) that quantifies the phenotype of CS PZ PCa.

\section{Materials and methods}

\section{Patient data}

This study was institutional review board approved, and all patients provided informed consent for the original dataset creation. The data used for this study was originally part of the ProstateX dataset [23]. A total of 206 patients from this dataset were scanned at the Radboud University Medical 
Center (Nijmegen, the Netherlands) in 2012, and these patients comprised the present study population. Patients in the ProstateX dataset had a median PSA level of $13 \mathrm{ng} / \mathrm{ml}$ (range 1 to $56 \mathrm{ng} / \mathrm{ml}$ ) with a median age of 66 (range 48 to 83 years) [24]. The mpMRI protocol was performed on a 3.0-T MRI scanner (MAGNETOM Trio or Skyra, Siemens Healthcare); see Table 1 for a summary of applied sequences (more detailed information can be found in the previously published challenge) [25]. All patients in this study underwent mpMRI of the prostate because of at least one previous negative systematic TRUS prostate biopsy and persistent clinical suspicion of PZ PCa (i.e., elevated PSA and/or abnormal DRE). These patients had a total of 262 prospectively called PI-RADS 3$5 \mathrm{PZ}$ lesions that were subsequently subjected to in-bore MRI targeted biopsy, which was used as reference standard (all under the supervision of a highly experienced radiologist in prostate mpMRI, $>20$ years of experience). PZ lesions with a Gleason score of $>6$ (International Society of Urological Pathology (ISUP) grade group $\geq 2$ ) were defined and labeled as CS PCa, while PZ lesions with a Gleason score of $\leq 6$, with normal or benign histopathology results (e.g., prostatitis, benign prostatic hyperplasia, or prostatic intraepithelial neoplasia), were labeled as the non-CS category.

\section{Training and test dataset}

Radiomics features [16] for the training dataset were calculated from prostate mpMRI scans of 130 patients who had a total of 171 prospectively called PI-RADS 3-5 PZ lesions, of which 35 proved to be CS PZ PCa and 136 were grouped in the non-CS PZ category according to MRI targeted biopsy results. Importantly, the test data set (which consisted of 76 patients with 91 prospectively called PI-RADS 3-5 PZ lesions, of which 20 were CS PCa and 71 were non-CS PZ entities) was kept separate from the training set and remained untouched until the development of the model, to avoid a biased result [26].

\section{Auto-fixed segmentation}

An auto-fixed tumor VOI was used for the extraction of the radiomics features in order to increase their reproducibility and robustness [27]. By using identical VOIs placed in the same manner, observer variability and dependency can be reduced. Originally, the prospectively called PI-RADS 3-5 PZ lesions were marked with a pin point in the visually most aggressive part of the lesion (area with the lowest apparent diffusion coefficient (ADC) value). Marking of this visually most aggressive part of the PZ lesion was performed under the supervision of an expert prostate radiologist ( $>20$ years of experience). Future clinical implementation of a model with auto-fixed segmentation requires the user to manually perform the marking. Scanner coordinates corresponding with the supervised marking were stored and converted to image coordinates. In this study, we then automatically created a spherical VOI with the lesion image coordinates at its center. The raster geometry package (Python Software Foundation) was used for the spherical volume calculation. For each of the image directions, a radius was calculated based on VOI size and image voxel spacing. The auto-fixed VOI size was set to $12 \mathrm{~mm}$ in order to sufficiently cover most prostate lesions which have an average diameter of $10 \mathrm{~mm}$ [28]. For a number of patients in the ProstateX dataset, deviations were discovered from the dimensional information reported in Table 1. Interpolation of these voxels was omitted due to uncertainty about the interpolation size and technique for mpMRI [29]. Solving these uncertainties for each mpMRI sequence requires a large number of experiments which is outside the scope of the current article. Additionally, no issues were expected due to the equal representation of the deviations in both the training and test datasets and the fact that feature calculation was based on a collection of voxels.

Table 1 Summary of sequences used for mpMRI of the prostate

\begin{tabular}{|c|c|c|c|}
\hline Sequence & $\begin{array}{l}\text { T2-weighted imaging } \\
\text { Turbo spin echo }\end{array}$ & $\begin{array}{l}\text { Dynamic contrast-enhanced imaging } \\
\text { 3D turbo gradient echo }\end{array}$ & Diffusion-weighted single-shot echo-planar imaging \\
\hline In-plane resolution (mm) & 0.5 & 1.5 & 2 \\
\hline Slice thickness (mm) & 3.6 & 4 & 3.6 \\
\hline Temporal resolution (s) & & 3.5 & \\
\hline Sequence orientation & Axial, sagittal, and coronal & Axial & Axial \\
\hline Additional remarks & No endorectal coil & $\begin{array}{l}\text { No endorectal coil } \\
\text { Used for K-trans calculation }\end{array}$ & $\begin{array}{l}\text { No endorectal coil } \\
b \text {-values of } 50,400, \text { and } 800 \mathrm{~s} / \mathrm{mm}^{2} \\
\text { Used for calculated } b \text {-value of } 1400 \mathrm{~s} / \mathrm{mm}^{2} \\
\text { and mono-exponentially calculated apparent } \\
\text { diffusion coefficient map }\end{array}$ \\
\hline
\end{tabular}

mpMRI multiparametric MRI 


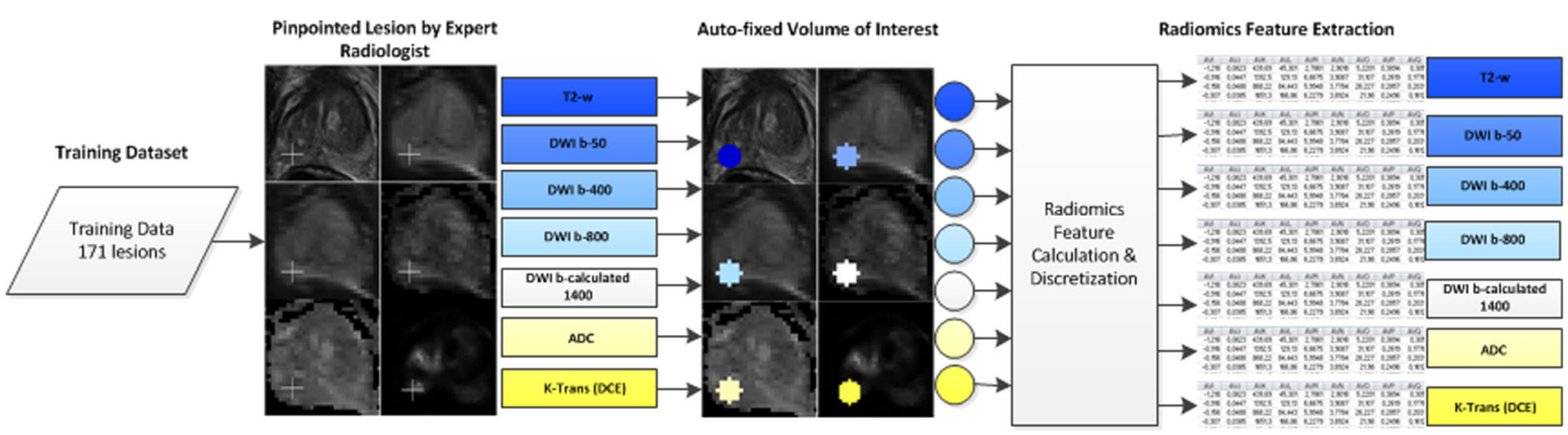

Fig. 1 Schematic pipeline for the extraction of radiomics features from mpMRI data. ADC $=$ apparent diffusion coefficient map, DCE $=$ dynamic contrast-enhanced, DWI = diffusion-weighted imaging, $\mathrm{T} 2-\mathrm{w}=\mathrm{T} 2$ weighted

\section{Radiomics features extraction}

Ninety-two quantitative radiomics features which comprised six different feature types were calculated in Python using Pyradiomics [30]. Eighteen first-order features which use basic statistics to characterize the voxel intensity distribution, 23 gray level co-occurrence matrix features (GLCM), 16 gray level run length matrix features (GLRLM), 16 gray level size zone matrix features (GLSZM), 14 gray level dependence matrix features (GLDM), and 5 neighboring gray tone difference matrix features (NGTDM) were used to quantify the image texture in the VOI. Previous work by Aerts et al and Zwanenburg et al provide full feature names and their mathematical descriptions [16, 29]. Pixels used for the calculation of the 80 texture features were discretized in fixed gray level bins (for further details, see supplemental digital content 1). An overview of the radiomics feature extraction pipeline is given in Fig. 1.

\section{Extreme gradient boosting, expert-based feature pre-selection, and the use of filters}

Due to the uncertain complimentary role of DCE imaging for the diagnosis of CS PCa [31, 32], an additional analysis was performed to determine the effect of DCE features in the radiomics approach. A total of two different mpMRI training datasets were created (Table 2). The first mpMRI dataset consisted of T2-weighted (T2-w) imaging, diffusionweighted imaging (DWI) with $b$-values of $50,400,800$, calculated $1400 \mathrm{~s} / \mathrm{mm}^{2}$, and an calculated ADC map (abbreviated as $\mathrm{T} 2-\mathrm{w}+\mathrm{DWI})$. The second mpMRI dataset expanded on this with DCE imaging, k-trans (abbreviated as T2-w + DWI + DCE). For each of the two mpMRI training datasets, two radiomics models were created. One of these models used a previously suggested machine learning approach for radiomics [22] with a combination of univariate feature selection and RF classifiers. In an effort to improve this, we first introduced another model based on a combination of multivariate feature selection and XGB classifiers. This can be considered a good fit for high-dimensional tabular data like in radiomics $[20,21]$. Both univariate and multivariate feature selection aim to find features with strong relationships with the output labels (CS PCa, non-CS entities). Multivariate feature selection also takes relationships between features into account. Detailed information about the machine learning approach can be found in supplemental digital content 2 . Second, we investigated whether expert-based feature preselection could increase the performance of the radiomics model [27]. Feature selection was performed by a specialized uro-radiologist (D.Y.) with 5 years of experience in mpMRI of the prostate. The selection was based on clinical experience and domain knowledge [33]; selected quantitative features were thought to correspond to clinical characteristics of CS PZ PCa or the non-CS category. Third, we investigated whether the use of image filters (e.g., edge enhancement and voxel intensity enhancement) improved the diagnostic accuracy of our model. Previous research has shown that applying certain image filters before feature extraction can enhance certain lesion type differences and improve diagnosis [34-38].

Table 2 Summary of mpMRI dataset composition

mpMRI dataset 1

T2-weighted imaging (axial, sagittal, and coronal planes)

Diffusion-weighted imaging

( $b$-values of 50, 400, 800, and calculated $b$-value of $1400 \mathrm{~s} / \mathrm{mm}^{2}$ )

Calculated ADC map
mpMRI dataset 2



$A D C$ apparent diffusion coefficient map, $D C E$ dynamic contrast-enhanced, $m p M R I$ multiparametric MRI 
Detailed filter descriptions and their effect can be found in supplemental digital content 3 . Using the best combination of mpMRI dataset (T2-w + DWI vs. T2-w + DWI + DCE), machine learning approach (RF vs. XGB), with or without expert-based feature pre-selection, and the effect of features taken from filtered images (e.g., edge enhancement), different models were created.

\section{Statistical analysis}

Each developed model was used to create an area under the curve (AUC) score based on $10 \times 10$-fold receiver operating curves (ROCs) on the training data. Training AUCs were checked for normality using Shapiro-Wilk's test and compared using the Wilcoxon signed rank test [39, 40]. Additionally, all models from the different experiments were evaluated on the separate test dataset. ROCs were created with corresponding AUCs and 95\% confidence intervals (CI) created with 5000 times bootstrapping. AUCs were compared using 5000 times bootstrapping. Statistical analyses were performed using R version 3.5.2 software (R Foundation for Statistical Computing) with the pROC package [41].

\section{Results}

\section{Effect of DCE on radiomics}

The comparison of models based on the two different mpMRI datasets (T2-w + DWI vs. T2-w + DWI + DCE) showed that the addition of DCE imaging did lead to a significant improvement on the training dataset $(p<0.001$, Table 3 ). This significant improvement found in the training dataset did not translate to the test dataset for both RF and XGB (AUC 0.780 vs. $0.745 p=0.657$, AUC 0.870 vs. $0.816 p=0.119$ ). ROCs for the test dataset of the models are given in Fig. 2, with corresponding AUCs in Table 5. The best scoring model from Table 5, AUC 0.870 (95\% CI 0.980-0.754), sensitivity 0.86 (63/73), and specificity 0.73 (11/15), takes a shared first place when compared to the original 71 entries and the over 200 ongoing entries in the ProstateX challenge [23, 42], which was the original purpose of the data used in this study. Figure 3 gives an evaluation example for model 3 (XGB + T2-w + DWI, AUC: 0.816, sensitivity 0.75 (55/73), and specificity $0.67(10 / 15))$ which was predicted correctly while Fig. 4 shows an example of a false positive.

\section{Multivariate selection and XGB versus univariate selection and $\mathbf{R F}$}

For both the mpMRI datasets defined in Table 2, the combination of multivariate feature selection and an XGB classifier achieved significantly higher AUCs when compared to univariate 
Fig. 2 Test dataset receiver operating curves (ROCs) for models 1 to 4 based on mpMRI dataset $1(\mathrm{~T} 2-\mathrm{W}+\mathrm{DWI})$ and mpMRI dataset $2(\mathrm{~T} 2-\mathrm{w}+\mathrm{DWI}+$ DCE). Model 1 (blue, mpMRI dataset 1) and model 2 (green, mpMRI dataset 2) curves are created by a combination of univariate feature selection and a random forest (RF) classifier. The curves for model 3 (red, mpMRI dataset 1) and model 4 (cyan, mpMRI dataset 2) were created using multivariate feature selection and extreme gradient boosting (XGB)

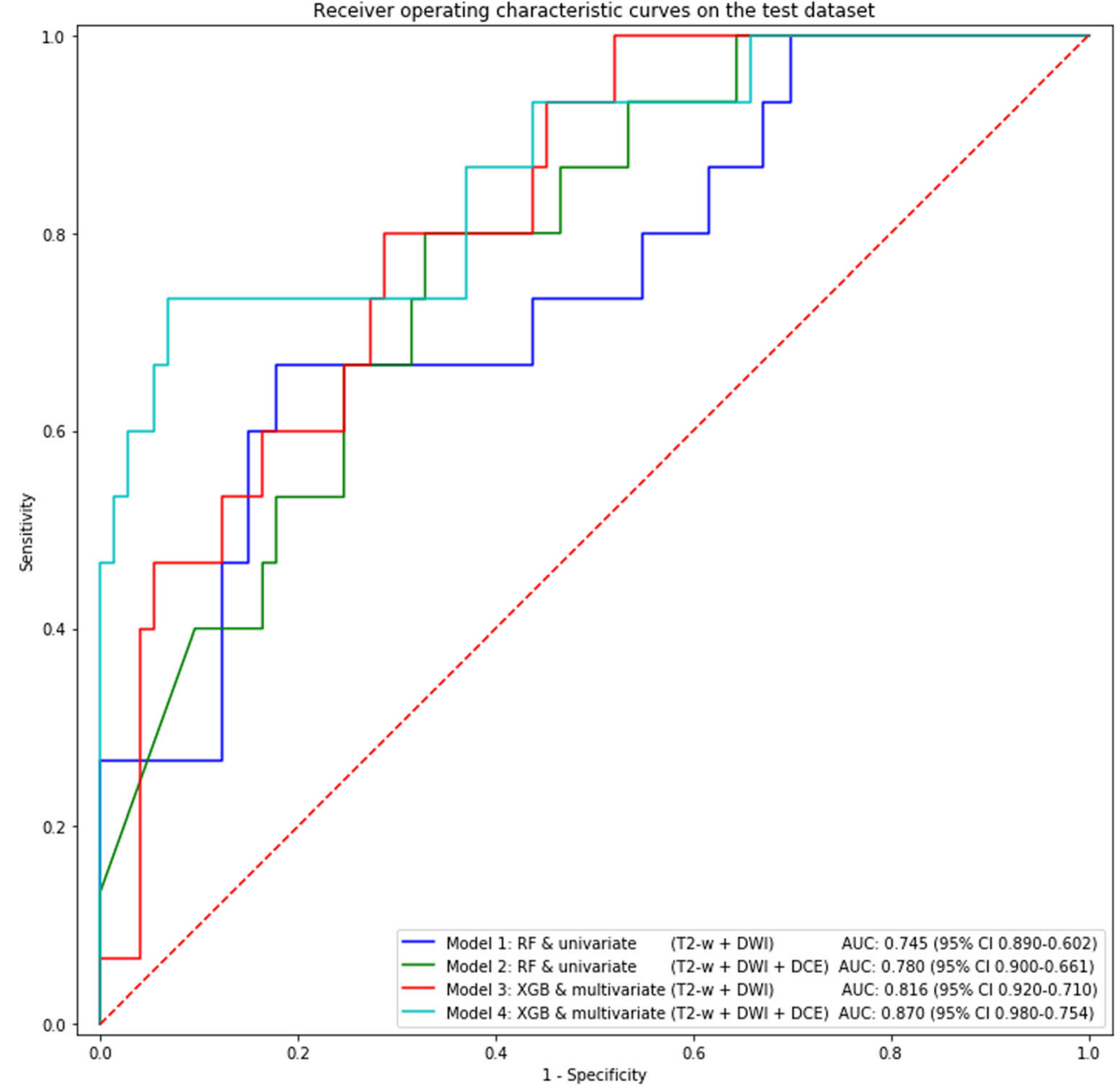



Fig. 3 True-positive example for model 3 (T2-w + DWI) which predicted a clinically significant (CS) prostate cancer (PCa) lesion. This patient had a peripheral zone (PZ) lesion (classifiable as PI-RADS 4) which was pinpointed (the visually most aggressive part) originally by an expert (arrow, first row), which proved to be CS PCa (Gleason score $>6$ ). a
T2-w (axial), b ADC, c DWI $b$-value $800 \mathrm{~s} / \mathrm{mm}^{2}$, d DWI calculated $b$ value $1400 \mathrm{~s} / \mathrm{mm}^{2}$. Second row, segmentations using the auto-fixed volume of interest (VOI, marked in white) were placed around the visually most aggressive lesion pinpoint 


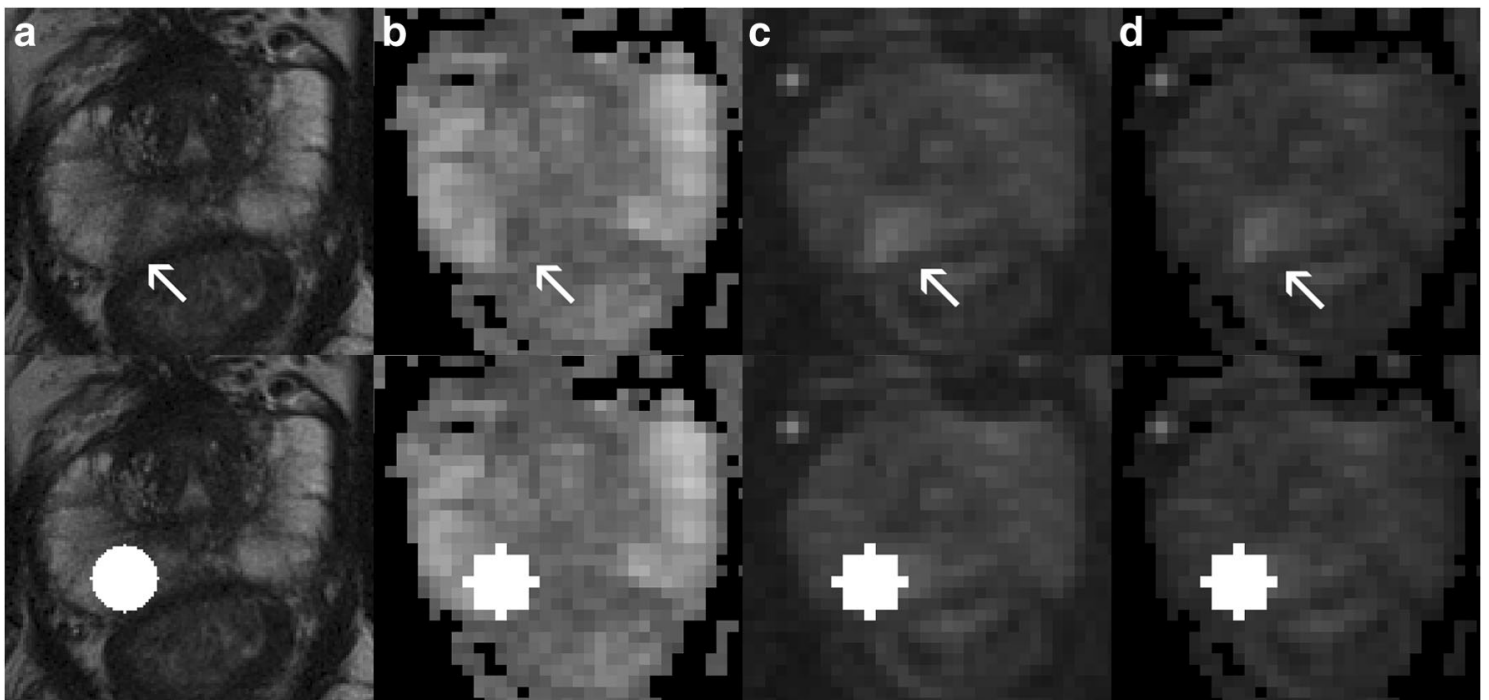

Fig. 4 False-positive example for model 3 (T2-w + DWI) which predicted a clinically significant $(\mathrm{CS})$ prostate cancer $(\mathrm{PCa})$ lesion. This patient had a peripheral zone (PZ) lesion (classifiable as PI-RADS 4) which was pinpointed (the visually most aggressive part) originally by an expert (arrow, first row), which proved to be a non-CS entity (Gleason score < 6). a T2-w (axial), b ADC, c DWI $b$-value $800 \mathrm{~s} / \mathrm{mm}^{2}$, d DWI calculated $b$-value $1400 \mathrm{~s} / \mathrm{mm}^{2}$. Second row, segmentations using the auto-fixed volume of interest (VOI, marked in white) were placed around the visually most aggressive lesion pinpoint

with an auto-fixed VOI, and achieved a relatively high AUC of 0.870 (95\% CI $0.980-0.754$ ) in the test dataset. Nevertheless, we found that the addition of features from DCE did not lead to a significantly improved radiomics model compared to features taken from $\mathrm{T} 2-\mathrm{w}$ and DWI alone. Furthermore, a combination of multivariate feature selection and XGB was found to be the best machine learning approach, while expert-based feature pre-selection and the addition of features taken from filtered images did not lead to a significant improvement. Importantly, we used datasets with prospectively called PI-RADS 3-5 lesions, in which the overall detection rate of CS PCa is known to be only 55\% [12]. Therefore, our results indicate that the developed model may provide additional diagnostic value and might potentially reduce the number of unnecessary biopsies.

Interestingly, we found that the addition of features taken from DCE imaging did not lead to a significant increase in diagnostic test performance $(p=0.119)$. This is in line with and supports the current trend of omitting DCE imaging from the routine MRI protocol and using the so-called biparametric MRI (bpMRI) to decrease study time and costs [43]. For routine prostate examinations, there is no difference in diagnostic performance between mpMRI and bpMRI [31, 32]. However, our results show a non-significant increase in diagnostic performance for models that did include DCE features. This nonsignificant increase might be explained using PI-RADSv2.1 which identifies five special patient scenarios where mpMRI should be preferred over bpMRI [44]. Our results also show that multivariate feature selection and XGB should be preferred over univariate feature selection and an RF classifier

(AUC of the latter, 0.780 (95\% CI 0.900-0.661), $p=0.028$ ).
Our best scoring model uses a combination of mpMRI features taken from T2-w, DWI, and DCE imaging, extracted 


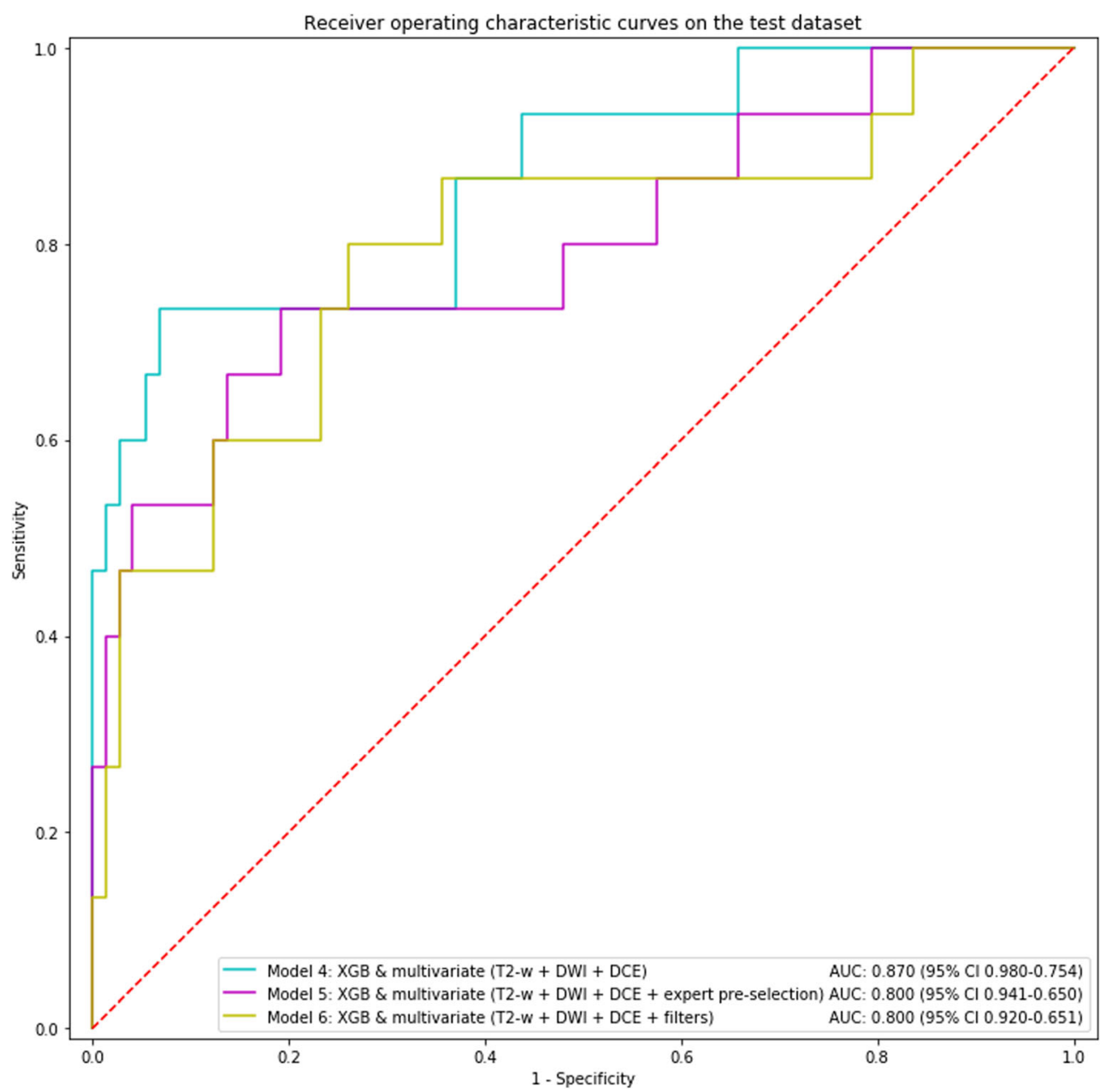

Fig. 5 Test dataset ROCs for model 4 (cyan, mpMRI dataset 2, T2-w + DWI + DCE and repeated from Fig. 2), model 5 (magenta, T2-w + DWI + DCE + expert pre-selection), and model 6 (yellow, T2-w + DWI + DCE + filters (supplemental digital content 3)) based on multivariate selection and XGB

This contradicts the results of a previous study by Parmar et al [22] that reported univariate feature selection and an RF classifier to be the best machine learning approach for radiomics [22]. This contradiction may be due to the different data types used, since Parmar et al [22] used computed tomography instead of mpMRI. Furthermore, our results showed that the univariate feature selection tends to focus on a single sequence, suggesting a good correlation between the single sequence of concern and the differentiation between CS PZ PCa and non-CS entities. However, given the fact that multivariate selection performed significantly better and did not focus on a single sequence, it appears that feature redundancies between features taken from a single sequence that are not tested in univariate selection diminish the performance of the model. Including expert-based pre-selection of radiomics features did not lead to a significant change in performance (AUC 0.800 (95\% CI 0.941-0.650), $p=0.273$ ). Though interestingly, it did lead to the least difference between the training and test datasets. A possible explanation for this finding may be that pre-selection based on clinical experience and domain knowledge eliminated the least reproducible features [27]. However, due to the loss in performance on the training dataset, the approach in which a single radiologist selects features based on experience and knowledge might not be viable and more research should be performed. The inclusion of features extracted from filtered mpMRI images, which should theoretically enhance lesion differences, did not significantly improve results (AUC 0.800 (95\% CI 0.920-0.651), $p=$ $0.177)$. This finding is in contrast to previous studies [35, $37,45]$ and may be explained by the use of a broad selection of multiple filter types while relying on the feature selection algorithms rather than domain knowledge. However, further investigation is needed before fully dismissing them.

There are a number of other studies that aimed to build an mpMRI radiomics model that quantifies the phenotype of CS PZ PCa $[18,19,46]$. Although it is difficult to fully compare the quantitative features we found with earlier research, e.g., due to different patient populations and variations in imaging protocols, some comparison between the present results and previous studies can be made. A recent study by Bonekamp et al [19] 


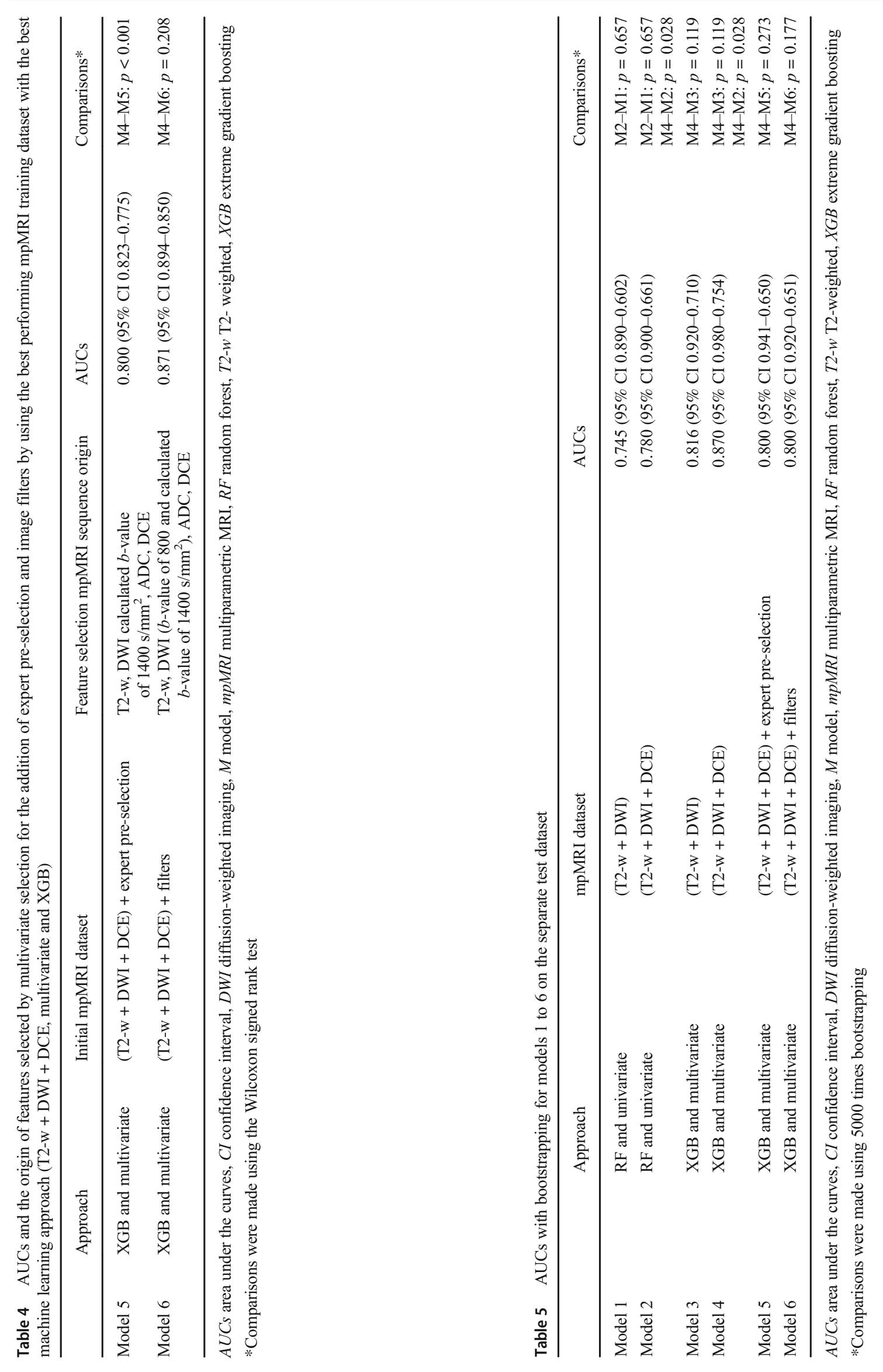


compared a radiomics model with the mean $\mathrm{ADC}$ and radiologist assessment for the diagnosis of CS PCa lesions. However, the approach used for the development of their radiomics model was limited by manual tumor lesion delineation and mixing of both PZ and TZ lesions. Not unimportantly, quantitative ADC measurements have a limited role in clinical practice. This is due to the variety of acquisition and analysis methodologies that do not allow for comparison of ADC values between centers and establishment of universally useful diagnostic cut-off values [47, 48]. Furthermore, manually delineating tumor boundaries is prone to making results observer dependent (besides being labor intensive) and mixing PZ and TZ lesions ignores the fact that both types of $\mathrm{PCa}$ are phenotypically different $[19,49-51]$. Another study by Khalvati et al [18] proposed a radiomics model which used a set of radiomics features with some statistical and textural features that partly matched our selection. Nevertheless, they did not investigate all mpMRI sequences such as DCE imaging and validated their radiomics model on a very small dataset of only 30 patients again without separating PZ from TZ lesions. Finally, a study by $\mathrm{Xu}$ et al introduced a radiomics model based on bpMRI radiomics features and a small set of clinical parameters [46]. Besides identical limitations to the ones mentioned above (manual delineation, mixing $\mathrm{PZ}$ and $\mathrm{TZ}$ lesions), $\mathrm{Xu}$ et al created a test dataset based on the date of the study instead of a random division. This, in combination with the observation that their test scores were higher than the then training scores, raises bias concerns. Additionally, they did not include a high calculated $b$-value which we found to be essential for models 1,3 , and 4 (Table 3).

The present study had several limitations. First, its results are only applicable to PZ lesions, and the model does not hold up for lesions in the TZ. TZ lesions, which are phenotypically different [49-51], should be investigated separately with the use of a dedicated model. Second, our study focused on lesion characterization and not on automatic detection of lesions suspicious of $\mathrm{PCa}$. A recently published study [52] investigated an automatic detection system for $\mathrm{PCa}$ lesions prior to a radiologist's interpretation. The authors of that study concluded that such a system introduced more false positives than a radiologist [52]. This raises the question of whether such automatic detection systems are suited for clinical practice at the moment. Third, due to the retrospective nature of the present study, mpMRI protocols were heterogeneous and performed on two different MRI systems. On the other hand, these differences yielded more diverse data that may actually have helped to increase reproducibility of the radiomics features [27]. Nevertheless, to be able to say with certainty that the model, and by extension the set of quantifying radiomics features, exhibit proper generalization, external validation should be performed in future studies. Finally, all patients underwent inbore MRI targeted biopsy, whereas prostatectomy may have served as a better reference standard. However, this reflects clinical practice, and only including patients who had undergone prostatectomy could have introduced selection bias [53].
In conclusion, the phenotype of CS PZ PCa lesions can be quantified using a radiomics approach based on features extracted from T2-w + DWI using an auto-fixed VOI. Although DCE features improve diagnostic performance, this is not statistically significant. Multivariate feature selection and XGB should be preferred over univariate feature selection and RF. The developed model may be a valuable addition to traditional visual assessment in diagnosing CS PZ PCa.

Acknowledgments The authors would like to thank Maarten Schellevis for his help with the test results.

Funding information The authors state that this work has not received any funding.

\section{Compliance with ethical standards}

Guarantor The scientific guarantor of this publication is Derya Yakar MD PhD.

Conflict of interest The authors declare that they have no competing interests.

Statistics and biometry No complex statistical methods were necessary for this paper.

Informed consent Written informed consent was not required for this study because it was retrospective research with data from an open public source.

Ethical approval Institutional review board approval was obtained.

Study subjects or cohorts overlap Some study subjects or cohorts have been previously reported in publications related to the ProstateX challenge. Litjens G, Debats O, Barentsz J, et al Computer-Aided Detection of Prostate Cancer in MRI. IEEE Trans. Med. Imaging. 2014;33(5):1083-1092. Our study used a very different approach with a different purpose.

\section{Methodology \\ - retrospective \\ - experimental \\ - performed at one institution}

Open Access This article is distributed under the terms of the Creative Commons Attribution 4.0 International License (http:// creativecommons.org/licenses/by/4.0/), which permits unrestricted use, distribution, and reproduction in any medium, provided you give appropriate credit to the original author(s) and the source, provide a link to the Creative Commons license, and indicate if changes were made.

\section{References}

1. Siegel RL, Miller KD, Jemal A (2018) Cancer statistics, 2018. CA Cancer J Clin 68:7-30

2. European Union (2018) European Cancer Information System. https://ecis.jrc.ec.europa.eu. Accessed 4 Jun 2018 
3. van den Bergh R, Loeb S, Roobol MJ (2015) Impact of early diagnosis of prostate cancer on survival outcomes. Eur Urol Focus 1:137-146

4. Harvey P, Basuita A, Endersby D, Curtis B, Iacovidou A, Walker M (2009) A systematic review of the diagnostic accuracy of prostate specific antigen. BMC Urol 9:1-9

5. Pokorny MR, De Rooij M, Duncan E et al (2014) Prospective study of diagnostic accuracy comparing prostate cancer detection by transrectal ultrasound-guided biopsy versus magnetic resonance (MR) imaging with subsequent mr-guided biopsy in men without previous prostate biopsies. Eur Urol 66:22-29

6. Loeb S, Vellekoop A, Ahmed HU et al (2013) Systematic review of complications of prostate biopsy. Eur Urol 64:876-892

7. Oberlin DT, Casalino DD, Miller FH, Meeks JJ (2017) Dramatic increase in the utilization of multiparametric magnetic resonance imaging for detection and management of prostate cancer. Abdom Radiol (NY) 42:1255-1258

8. Thompson JE, Van Leeuwen PJ, Moses D et al (2016) The diagnostic performance of multiparametric magnetic resonance imaging to detect significant prostate cancer. J Urol 195:1428-1435

9. Ahmed HU, El-Shater Bosaily A, Brown LC et al (2017) Diagnostic accuracy of multi-parametric MRI and TRUS biopsy in prostate cancer (PROMIS): a paired validating confirmatory study. Lancet 389:815-822

10. Weinreb JC, Barentsz JO, Choyke PL et al (2016) PI-RADS Prostate Imaging - reporting and data system: 2015, Version 2. Eur Urol 69:16-40

11. Kasel-Seibert M, Lehmann T, Aschenbach R et al (2016) Assessment of PI-RADS v2 for the detection of prostate cancer. Eur J Radiol 85:726-731

12. Hofbauer SL, Kittner B, Maxeiner A et al (2018) Validation of Prostate Imaging Reporting and Data System version 2 for the detection of prostate cancer. J Urol 200:767-773

13. van der Leest M, Cornel E, Israël B et al (2018) Head-to-head comparison of transrectal ultrasound-guided prostate biopsy versus multiparametric prostate resonance imaging with subsequent magnetic resonance-guided biopsy in biopsy-naïve men with elevated prostate-specific antigen: a large prospective mu. Eur Urol 5:579581

14. Rouvière $\mathrm{O}$, Puech $\mathrm{P}$, Renard-Penna R et al (2018) Use of prostate systematic and targeted biopsy on the basis of multiparametric MRI in biopsy-naive patients (MRI-FIRST): a prospective, multicentre, paired diagnostic study. Lancet Oncol 20:100-109

15. Fei B (2017) Computer-aided diagnosis of prostate cancer with MRI. Curr Opin Biomed Eng 3:20-27

16. Aerts HJWL, Velazquez ER, Leijenaar RTH et al (2014) Decoding tumour phenotype by noninvasive imaging using a quantitative radiomics approach. Nat Commun 5:4006

17. Cameron A, Khalvati F, Haider MA, Wong A (2016) MAPS: a quantitative radiomics approach for prostate cancer detection. IEEE Trans Biomed Eng 63:1145-1156

18. Khalvati F, Zhang J, Chung AG, Shafiee MJ, Wong A, Haider MA (2018) MPCaD: a multi-scale radiomics-driven framework for automated prostate cancer localization and detection. BMC Med Imaging 18:16

19. Bonekamp D, Kohl S, Wiesenfarth M et al (2018) Radiomic machine learning for characterization of prostate lesions with MRI: comparison to ADC values. Radiology 289:128-137

20. Chen T, Guestrin C (2016) XGBoost: a scalable tree boosting system. KDD 16 Proceedings of the 22nd ACM SIGKDD International Conference on Knowledge Discovery and Data Mining. San Francisco, California, USA 785-794 https://doi.org/ $10.1145 / 2939672.2939785$
21. Bennasar M, Hicks Y, Setchi R (2015) Feature selection using joint mutual information maximisation. Expert Syst Appl 42:8520-8532

22. Parmar C, Grossmann P, Bussink J, Lambin P, Aerts HJWL (2015) Machine learning methods for quantitative radiomic biomarkers. Sci Rep 5:1-11

23. Litjens G, Debats O, Barentsz J, Karssemeijer N, Huisman H (2017) SPIE-AAPM PROSTATEx challenge data. In: Cancer Imaging Arch. https://wiki.cancerimagingarchive.net/display/ Public/SPIE-AAPM-NCI+PROSTATEx+Challenges. Accessed 1 Jun 2018

24. Armato SG Jr, Huisman H, Drukker K et al (2018) PROSTATEx challenges for computerized classification of prostate lesions from multiparametric magnetic resonance images. J Med Imaging (Bellingham) 5:044501

25. Litjens G, Debats O, Barentsz J, Karssemeijer N, Huisman H (2014) Computer-aided detection of prostate cancer in MRI. IEEE Trans Med Imaging 33:1083-1092

26. Smialowski P, Frishman D, Kramer S (2009) Pitfalls of supervised feature selection. Bioinformatics 26:440-443

27. Kumar V, Gu Y, Basu S et al (2012) Radiomics: the process and the challenges. Magn Reson Imaging 30:1234-1248

28. Wolters T, Roobol MJ, Van Leeuwen PJ et al (2011) A critical analysis of the tumor volume threshold for clinically insignificant prostate cancer using a data set of a randomized screening trial. J Urol 185:121-125

29. Zwanenburg A, Leger S, Vallières M, Löck S (2016) Image biomarker standardisation initiative. ArXiv ID: 1612.07003

30. Van Griethuysen JJM, Fedorov A, Parmar C et al (2017) Computational radiomics system to decode the radiographic phenotype. Cancer Res 77:e104-e107

31. Barth BK, De Visschere PJL, Cornelius A et al (2017) Detection of clinically significant prostate cancer: short dual- pulse sequence versus standard multiparametric MR imaging - a multireader study Radiology 284:725-736

32. Junker D, Steinkohl F, Fritz V et al (2018) Comparison of multiparametric and biparametric MRI of the prostate: are gadolinium-based contrast agents needed for routine examinations? World J Urol. https://doi.org/10.1007/s00345-018-2428-y

33. Guyon I, Elisseeff A (2003) An introduction to variable and feature selection. J Mach Learn Res 3:1157-1182

34. Chen JS, Huertas A, Medioni G (1987) Fast convolution with Laplacian-of-Gaussian masks. IEEE Trans Pattern Anal Mach Intell PAMI-9:584-590

35. Thawani R, McLane M, Beig N et al (2018) Radiomics and radiogenomics in lung cancer: a review for the clinician. Lung Cancer 115:34-41

36. Bartušek K, Přinosil J, Smékal Z (2011) Wavelet-based de-noising techniques in MRI. Comput Methods Programs Biomed 104:480 488

37. Ojala T, Pietikäinen M, Harwood D (1996) A comparative study of texture measures with classification based on featured distributions. Pattern Recognit 29:51-59

38. Barkan O, Weill J, Wolf L, Aronowitz H (2013) Fast high dimensional vector multiplication face recognition. In: 13 Proceedings of the IEEE International Conference on Computer Vision (ICCV), (2013) December, pp. 1960-1967 https://doi.org/10.1109/ICCV. 2013.246

39. Shapiro ASS, Wilk MB (1965) An Analysis of Variance Test for Normality (Complete Samples). Biometrika 52:591-611

40. Wilcoxon F (1946) Individual comparisons of grouped data by ranking methods. J Econ Entomol 39:269

41. Robin X, Turck N, Hainard A et al (2011) pROC: an open-source package for $\mathrm{R}$ and $\mathrm{S}+$ to analyze and compare ROC curves. BMC Bioinformatics 12:77 
42. Radboudumc (2018) ProstateX grand challenge. https://prostatex. grand-challenge.org/. Accessed 7 Feb 2019

43. Sackett J, Choyke PL, Turkbey B (2019) Prostate imaging reporting and data system version 2 for MRI of prostate cancer: can we do better? AJR Am J Roentgenol 212:1-9

44. Turkbey B, Rosenkrantz AB, Haider MA et al (2019) Prostate imaging reporting and data system version 2.1: 2019 update of prostate imaging reporting and data system version 2. Eur Urol 0232:112

45. Ahonen T, Hadid A, Pietikäinen M (2004) Face recognition with local binary patterns. Comput Vis ECCV 2004:469-481

46. Xu M, Fang M, Zou J et al (2019) Using biparametric MRI radiomics signature to differentiate between benign and malignant prostate lesions. Eur J Radiol 114:38-44

47. DeSouza NM, Winfield JM, Waterton JC et al (2018) Implementing diffusion-weighted MRI for body imaging in prospective multicentre trials: current considerations and future perspectives. Eur Radiol 28:1118-1131

48. Sasaki M, Yamada K, Watanabe Y et al (2008) Variability in absolute apparent diffusion coefficient values across different platforms may be substantial: a multivendor, multi-institutional comparison study. Radiology 249:624-630
49. Ginsburg SB, Algohary A, Pahwa S et al (2017) Radiomic features for prostate cancer detection on MRI differ between the transition and peripheral zones: preliminary findings from a multiinstitutional study. J Magn Reson Imaging 46:184-193

50. Sakai I, Harada K, Hara I, Eto H, Miyake H (2005) A comparison of the biological features between prostate cancers arising in the transition and peripheral zones. BJU Int 96:528-532

51. Sakai I, Harada K, Kurahashi T, Yamanaka K, Hara I, Miyake H (2006) Analysis of differences in clinicopathological features between prostate cancers located in the transition and peripheral zones. Int J Urol 13:368-372

52. Greer MD, Lay N, Shih JH et al (2018) Computer-aided diagnosis prior to conventional interpretation of prostate mpMRI: an international multi-reader study. Eur Radiol 10:4407-4417

53. Wang NN, Fan RE, Leppert JT et al (2018) Performance of multiparametric MRI appears better when measured in patients who undergo radical prostatectomy. Res Rep Urol 10:233-235

Publisher's note Springer Nature remains neutral with regard to jurisdictional claims in published maps and institutional affiliations. 